\title{
Electroconvulsive Therapy for Parkinson's Disease with Depression and Neuroleptic Malignant Syndrome: A Case Report
}

\author{
Yuhei Mori, Itaru Miura, Michinari Nozaki, Yusuke Osakabe, Ryuta Izumi, Takahiro Akama, So Kimura, \\ Hirooki Yabe \\ Department of Neuropsychiatry, Fukushima Medical University School of Medicine, Fukushima, Japan
}

\begin{abstract}
Parkinson's disease is often complicated by psychiatric symptoms. Psychiatrists are caught in a dilemma between such symptoms and physical treatment since Parkinson's disease sometimes shows treatment resistance based on pharmacological treatment-induced dopamine dysfunction. Here, we report on a 64-year-old woman with a 15-year history of Parkinson's disease with stage IV severity based on the Hoehn and Yahr scale. She was admitted to our hospital with a diagnosis of major depressive disorder with psychotic features. Unfortunately, her treatment course for depression was complicated by neuroleptic malignant syndrome. Because we were concerned about the persistence of her depressive symptoms, the risk of psychotropic drugs causing adverse effects, and progressive disuse syndrome, we administered modified electroconvulsive therapy. Her symptoms of neuroleptic malignant syndrome and depression sufficiently improved after five sessions of modified electroconvulsive therapy. Additionally, the primary motor symptoms of her Parkinson's disease also markedly improved. The improvement of neuroleptic malignant syndrome and her motor symptoms based on dopamine dysfunction can be explained by electroconvulsive therapy's effectiveness in activating dopamine neurotransmission. Besides, the marked improvement of her depressive episode with psychotic features was presumed to involve dopamine receptor activation and regulation. Because advanced Parkinson's disease can sometimes be refractory to treatment based on pharmacological treatment-induced dopamine dysfunction, psychiatrists often have difficulty treating psychiatric symptoms; electroconvulsive therapy may stabilize the dopaminergic system in such cases, presenting a possible non-pharmacologic treatment option for Parkinson's disease.
\end{abstract}

KEY WORDS: Depression; Dopamine; Electroconvulsive therapy; Neuroleptic malignant syndrome; Parkinson disease.

\section{INTRODUCTION}

Parkinson's disease (PD) is often complicated by psychiatric symptoms as it progresses. Although we usually initiate psychopharmacological treatment with antidepressants and antipsychotics, PD poses a risk for physical complications caused by such drugs (e.g., worsening of motor symptoms, over sedation, aspiration pneumonia, neuroleptic malignant syndrome [NMS]). Therefore, we are often caught in a dilemma between psychiatric symptoms and physical treatment. Although electroconvulsive

Received: November 6, 2020 / Revised: December 29, 2020 Accepted: February 24, 2021

Address for correspondence: Yuhei Mori

Department of Neuropsychiatry, Fukushima Medical University School of Medicine, 1 Hikarigaoka, Fukushima city, Fukushima 960-1295, Japan

E-mail: yuhei-m@fmu.ac.jp

ORCID: https://orcid.org/0000-0001-9054-1692 therapy (ECT) has been used in patients with PD for the treatment of depression or motor symptoms, it remains difficult to determine the application of ECT in the clinical course of PD. Herein, we present the case of a woman with PD. She suffered from NMS during the treatment of depression and was treated with ECT. As a result, in addition to the improvement of NMS and depression, the primary motor symptoms significantly improved. We report this case as an examination of the effectiveness of ECT as a non-pharmacological therapy for PD. The patient and her family provided consent for publication of this case. This report obeyed the rule of the Ethics Committee of Fukushima Medical University Hospital, and conformed to the provisions of the Declaration of Helsinki.

(ㄷ) This is an Open-Access article distributed under the terms of the Creative Commons Attribution Non-Commercial License (http://creativecommons.org/licenses/by-nc/4.0) which permits unrestricted non-commercial use, distribution, and reproduction in any medium, provided the original work is properly cited. 


\section{CASE}

A 64-year-old woman treated for PD for 15 years had been suffering from progressive motor symptoms and had received $500 \mathrm{mg}$ of levodopa per day. Her severity was classified as stage IV according to the Hoehn and Yahr scale. She had experienced delusions and hallucinations, which improved by oral administration of donepezil, quetiapine, and valproic acid. However, shortly afterward, insomnia, loss of appetite, fatigue, and suicidal ideation appeared, which gradually worsened. Because she did not improve, she was admitted to our hospital with the diagnosis of major depressive disorder (single episode, severe, with psychotic features), according to the Diagnostic and Statistical Manual of Mental Disorders, Fifth Edition. At this point, the Montgomery Åsberg Depression Rating Scale score was 58 as shown in Figure 1. She had no progressive cognitive decline that indicated dementia. Although she was administered clomipramine intravenously and quetiapine (orally, gradually increased), her fever had been persistent for several days prior to admission. By day 3, she had a fever of approximately $38^{\circ} \mathrm{C}$. Blood sampling showed inflammatory findings, and a computed tomographic scan showed a consolidation in the lung field. Her condition was complicated by aspiration pneumonia, and she stopped eating. Consequently, antibiotics were started intravenously and she was administered all oral medications (including levodopa) through a nasogastric tube. Although her fever had resolved once previously, the patient again experienced a fever of approximately $38^{\circ} \mathrm{C}$ a few days after starting antibiotics. Additionally, marked muscle rigidity, autonomic system disorders (blood pressure changes, heavy sweating), salivation, and a delirium-like change in consciousness appeared. We diagnosed NMS based on these clinical symptoms, according to the criteria of Levenson [1] and Pope et al. [2]. At that point, her depression had not improved sufficiently. She attempted suicide by biting her tongue. Although her circulatory and respiratory states were stable, we were concerned about persistent depression, the risk of using psychotropic drugs, and progressive disuse syndrome due to prolonged bed rest. Therefore, we decided to apply modified electroconvulsive therapy (mECT) to improve her psychiatric symptoms immediately. In total, mECT was performed five times using the Somatics Thymatron ${ }^{\circledR}$ ECT machine. The Thymatron device settings were as follows: 0.9-A current, 0.5-millisecond pulse width, 30-Hz stimulation frequency, and 5.6-second stimulus duration. Electrographic seizure durations were 25 to 65 seconds during these treatments. As mECT proceeded, her autonomic system disorders disappeared, and her consciousness level improved. She no longer made depressive statements that were accompanied by suicidal thoughts, and there was a return to activity, in-

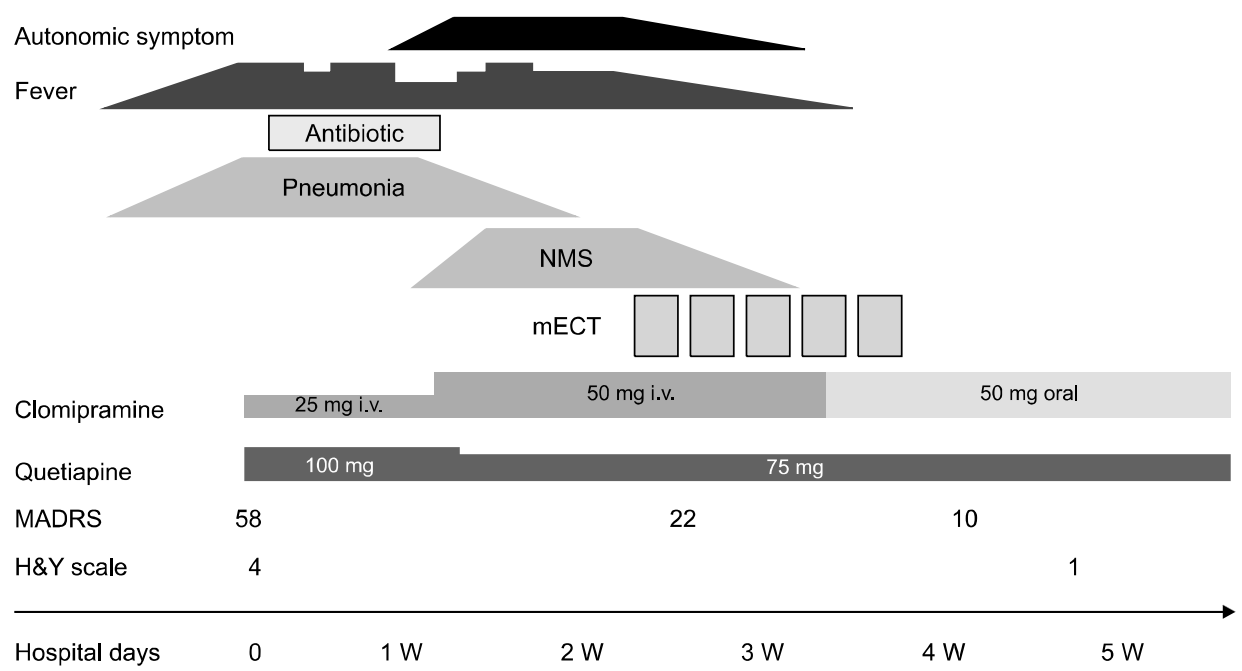

Fig. 1. Course of treatment and changes in several symptom scores. Hospital day 0 represents the day of admission. The MADRS score decreased after mECT. Similarly, the H\&Y scale, which reflects the degree of motor symptoms, improved.

H\&Y scale, Hoehn and Yahr scale; NMS, neuroleptic malignant syndrome; mECT, modified electroconvulsive therapy; i.v., intravenous; MADRS, Montgomery Åsberg Depression Rating Scale. 
cluding the ability to walk on her own, from a state in which she tended to lie down. She was able to eat well and sleep well at night. Therefore, NMS symptoms and depression sufficiently improved. As shown in Figure 1, the MARDS score improved to 10 . Furthermore, primary motor symptoms improved markedly to stage I of the Hoehn and Yahr scale. She had previously considered institutionalization due to severe motor symptoms; however, she was able to be discharged from our hospital and went home due to the significant improvement in motor symptoms. She has been living at home for approximately 1 year since discharge.

\section{DISCUSSION}

To the best of our knowledge, this is the first report evaluating the effectiveness of ECT on NMS, motor symptoms, and depression combined in patients with PD. NMS is known to be associated with central dopaminergic dysfunction induced by antipsychotics with D2 receptor antagonism [3]. Central dopaminergic systems are responsible for consciousness, temperature regulation, muscle tone, and movement. Blockade of these systems are involved in the symptoms of NMS. Neuroleptic-induced dopamine blockade in the nigrostriatal dopamine pathway causes rigidity, and dopamine blockade in the hypothalamus may explain the impairment of the autonomic as well as central thermoregulation. In this case, although there was no creatine kinase elevation and leukocytosis (which were characterized as NMS), the patient had other symptoms that satisfied the criteria of NMS [1,2]. Previous reports have shown NMS to be induced by low dopamine affinity quetiapine and the antidepressant clomipramine $[4,5]$, including NMS without elevated serum creatine kinase [6]. Therefore, we have concluded that NMS was associated with using quetiapine and/or clomipramine on the background of risk factors such as infection, dehydration, malnutrition, and original vulnerability of the dopamine nervous system. Although psychopharmacological treatment was limited due to the possibility of drug-induced NMS, rapid and reliable improvement of her psychiatric symptoms was needed because of severe depression accompanied by a suicide attempt. Therefore, we decided to apply $\mathrm{mECT}$ immediately; treatment brought about a remarkable improvement in NMS, depression, and motor symptoms. Previous studies have reported that
ECT resulted in an overall activation of dopamine neurotransmission [7]. The improvement of NMS symptoms (i.e., fever, muscle rigidity) which is caused by the blockade of central dopaminergic systems suggest that ECT might activate the dopamine neurotransmission in hypothalamus and striatum. Thus, in this case, the improvement of NMS and motor symptoms in dopamine dysfunction can be explained by the effectiveness of ECT in activating dopamine neurotransmission in hypothalamus and nigrostriatal pathway.

As regards depression with PD, it can be conceptualized as a hypodopaminergic state, since dopamine agonists exert antidepressant effects in animal models of depression [8]. Moreover, previous studies have reported that depression in PD was associated with decreased dopamine in the mesocortical dopamine pathway, in addition to the nigrostriatal dopamine pathway $[9,10]$. Therefore, we presumed that her depression was related to a hypodopaminergic state in the mesocortical dopamine pathway and the nigrostriatal dopamine pathway. Conversely, the effectiveness of antipsychotics for depression may suggest that depression is associated with hyperdopaminergic function, particularly in patients with psychotic depression. Since this patient's depression included psychotic features, the hyperdopaminergic state might exist in the mesolimbic dopamine pathway, although dopaminergic dynamics in depression with PD are very complicated. Previous studies have reported that repeated ECT might have an effect of density reduction on postsynaptic dopamine receptors, resulting in the improvement of psychosis [11]. We presumed that her marked improvement of depression with psychotic features involved regulation of dopamine receptors in the mesolimbic dopamine pathway. In other words, although her central dopaminergic system was in an imbalanced state, ECT might have contributed to the improvement in symptoms as a dopamine system stabilizer.

However, our report had some limitations. It is unclear whether the same therapeutic response could be obtained when her depression relapsed. PD is a progressive disease, which may lead to resistance to ECT treatment in the future. In addition, although we considered that the improvement of her symptoms is likely due to the effectiveness of ECT in several dopaminergic pathways, the possibility of the improvement by non-dopaminergic neural transmission (i.e., noradrenaline and serotonin) and other 
mechanisms of ECT cannot be denied [12,13].

In summary, ECT resulted in a remarkable simultaneous improvement in the NMS, depression, and motor symptoms of PD in this patient. If patients with PD have motor and neuropsychiatric symptoms such as depression, psychosis, and NMS with intolerance of psychopharmacological treatment, ECT may be a safe and effective treatment option. Moreover, ECT can show an early treatment response, which prevents disuse syndrome and enables patients to perform activities of daily living better. Since advanced PD sometimes shows resistance based on pharmacological treatment-induced dopamine dysfunction, psychiatrists often have difficulty treating psychiatric symptoms; ECT might have the ability to stabilize the dopaminergic system in such cases, which can sometimes open the treatment path for PD.

\section{Acknowledgments}

We appreciate the cooperation and time commitment of all team physicians.

\section{Conflicts of Interest}

No potential conflict of interest relevant to this article was reported.

\section{Author Contributions}

Yuhei Mori, Itaru Miura designed the report, contributed to interpretations of all results, and drafted the manuscript and figure. Yuhei Mori, Michinari Nozaki, Yusuke Osakabe, Ryuta Izumi, Takahiro Akama, and So Kimura treated the patient and discussed the treatment. Hirooki Yabe supervised the work. All authors reviewed the manuscript and approved for submission.

\section{ORCID}

Yuhei Mori Itaru Miura

Michinari Nozaki

Yusuke Osakabe

Ryuta Izumi

Takahiro Akama https://orcid.org/0000-0001-9054-1692

https://orcid.org/0000-0002-2133-5809

https://orcid.org/0000-0002-6235-2365

https://orcid.org/0000-0002-0886-2233

https://orcid.org/0000-0002-8453-3474

https://orcid.org/0000-0003-4677-5293
So Kimura

https://orcid.org/0000-0001-6925-7822

Hirooki Yabe

https://orcid.org/0000-0003-2668-129X

\section{REFERENCES}

1. Levenson JL. Neuroleptic malignant syndrome. Am J Psychiatry 1985; 142:1137-1145.

2. Pope HG Jr, Keck PE Jr, McElroy SL. Frequency and presentation of neuroleptic malignant syndrome in a large psychiatric hospital. Am J Psychiatry 1986;143:1227-1233.

3. Velamoor R. Neuroleptic malignant syndrome: a neuro-psychiatric emergency: recognition, prevention, and management. Asian J Psychiatr 2017;29:106-109.

4. Gortney JS, Fagan A, Kissack JC. Neuroleptic malignant syndrome secondary to quetiapine. Ann Pharmacother 2009;43: 785-791.

5. Haddow AM, Harris D, Wilson M, Logie H. Clomipramine induced neuroleptic malignant syndrome and pyrexia of unknown origin. BMJ 2004;329:1333-1335.

6. Nisijima K, Shioda K. A rare case of neuroleptic malignant syndrome without elevated serum creatine kinase. Neuropsychiatr Dis Treat 2014;10:403-407.

7. Cumper SK, Ahle GM, Liebman LS, Kellner CH. Electroconvulsive therapy (ECT) in Parkinson's disease: ECS and dopamine enhancement. J ECT 2014;30:122-124.

8. Breuer ME, Groenink L, Oosting RS, Buerger E, Korte M, Ferger $\mathrm{B}$, et al. Antidepressant effects of pramipexole, a dopamine D3/D2 receptor agonist, and 7-OH-DPAT, a dopamine D3 receptor agonist, in olfactory bulbectomized rats. EurJ Pharmacol 2009;616:134-140.

9. Vriend C, Pattij T, van der Werf YD, Voorn P, Booij J, Rutten $\mathrm{S}$, et al. Depression and impulse control disorders in Parkinson's disease: two sides of the same coin? Neurosci Biobehav Rev 2014;38:60-71.

10. Vriend C, Raijmakers $P$, Veltman DJ, van Dijk KD, van der Werf YD, Foncke EM, et al. Depressive symptoms in Parkinson's disease are related to reduced [123I]FP-CIT binding in the caudate nucleus. I Neurol Neurosurg Psychiatry 2014;85: 159-164.

11. Cooper SJ, Leahey W, Green DF, King DJ. The effect of electroconvulsive therapy on CSF amine metabolites in schizophrenic patients. Br J Psychiatry 1988;152:59-63.

12. Baldinger $P$, Lotan A, Frey R, Kasper S, Lerer B, Lanzenberger R. Neurotransmitters and electroconvulsive therapy. J ECT 2014;30:116-121.

13. Singh A, Kar SK. How electroconvulsive therapy works?: understanding the neurobiological mechanisms. Clin Psychopharmacol Neurosci 2017;15:210-221. 